\title{
Cupping in dermatology: a critical review and update
}

\author{
Yssra Soliman ${ }^{1}$, Nouran Hamed², Amor Khachemoune ${ }^{3,4} \bowtie$
}

\begin{abstract}
Cupping is an ancient procedure that has been practiced for thousands of years. It has been used to treat a variety of medical conditions, including dermatological ones. Cupping has been described in the literature for the treatment of conditions such as acne and eczema. The procedure is fundamentally divided into dry cupping and wet cupping; however, there have been many modern adaptations. Adverse events related to the procedure have been reported in the literature and should be considered by patients. However, cupping has a promising role in helping manage dermatological conditions.
\end{abstract}

Keywords: cupping, complementary and alternative medicine, eczema, acne, burns

Received: 12 October 2017 | Returned for modification: 5 February 2018 | Accepted: 26 February 2018

\section{Introduction}

Cupping is an ancient therapeutic procedure that has been practiced for thousands of years and throughout various civilizations (1). It dates as far back as ancient Egypt, around 1500 BC, where cupping was seen as a remedy for diseases including ocular inflammation, tonsil enlargement, headaches, gout, and lack of sleep (1). Drawings of cupping can be seen on the Temple of Kom Ombo near Luxor, one of the Egypt's most ancient cities $(2,3)$. The Ebers Papyrus, an Egyptian medical text, describes cupping used to treat various ailments including pain, fever, and dysmenorrhea.

In ancient Greece, cupping was described by Hippocrates (4). Hippocratic physicians used cupping to restore the balance between the four humors: blood, phlegm, yellow bile, and black bile (5). Galen, a Greek physician and philosopher, was known to use cupping as well (6).

In the Chinese tradition, cupping was described in the early Han dynasty $(7,8)$. They believed that with "acupuncture and cupping, more than half of the ills [are] cured" (8). There are records of cupping being used in treatment of patients with tuberculosis and chronic cough, and even to treat poisonous snakebites (8). Previously, animal horns were utilized in the procedure. Over time, the procedure evolved to use bamboo, earthenware, and, in modern times, glass cups.

It is evident that cupping was practiced in various forms throughout many civilizations. The two main types of cupping can simply be defined as "wet" or "dry" cupping. The fundamental difference is that wet cupping involves an element of bloodletting. During early Islamic times, wet cupping, called al-hijamah, was central to medicinal practices (9). Al-hijamah means "to reduce in size" or "to return the body to its natural state" (2). In the Islamic tradition, Prophet Mohammed said to his followers "indeed the best of remedies you have is hijamah (cupping)" (10). Al-hijamah was used in the prophetic tradition to cure headaches and pain, among other ailments.

Today, there is an increased interest in integrating complementary and alternative medicine (CAM) with current modern medical practices. Cupping has recently become evident in modern culture, especially after the 2016 Olympics in Rio de Janeiro, Brazil (11). Prominent athletes were seen competing with the dark circu- lar marks evident on their backs (12). Many competitive athletes have partially attributed their success to cupping because it reduces pain and improves recovery.

However, many medical providers may still be opposed to integrating CAM due to the limited evidence-based medicine supporting its benefits. Cupping is still used in different variations worldwide, particularly in the Middle East and in East Asian countries.

\section{Types of cupping}

There are many variations of cupping; most commonly they are characterized as dry cupping or wet cupping.

\section{Dry cupping}

Dry cupping is performed by coating a thick glass cup with alcohol (13). A flame is applied and, just before extinguishing it, the coated cup is applied to the patient's skin. It is important to note that the flame should heat the air in the cup and not the cup itself (14). As the cup is placed on the skin, negative pressure suctions the skin into the cup (15). The cups remain in place for about 1015 minutes (14). Patients are often left with circular ecchymosis and petechiae, due to trauma to superficial blood vessels (15).

\section{Wet cupping}

Wet cupping is different from dry cupping in that it involves bloodletting (16). It is thought that wet cupping may be more advantageous because it changes the patient's circulation in two ways (14). First, blood loss stimulates the bone marrow to produce new erythrocytes and leukocytes. Second, there is a reduction in blood viscosity, which may lead to improved blood flow.

There are two different forms of wet cupping that are commonly used (17). The first is referred to as the cupping, puncturing, and cupping method (CPC). It consists of six steps: demarcating the skin, sterilizing the area, cupping, puncturing, cupping, and sterilizing once again. The second method is the puncturing and cupping (PC) method, which involves five steps: demarcating the skin, sterilizing the area, puncturing, cupping, and sterilizing once again. 
The feature that differentiates CPC from PC is that the former essentially begins with dry cupping and is then followed by puncturing. The PC method starts with puncturing. The skin is punctured with a scarificator, an instrument with a series of small blades (14). When the scarificator is applied to the skin, the result is a series of small incisions that are approximately $1 \mathrm{~cm}$ long and $4 \mathrm{~mm}$ deep. The cups are then placed on top of these areas to extract blood through the lesions. Approximately 50 to $300 \mathrm{ml}$ can be extracted during each cupping session. CPC is used more widely in the Middle East and is traditionally known as al-hijamah $(17,18)$. The PC method is more commonly utilized in China, Korea, and Germany $(17,18)$.

\section{Adaptations to cupping}

There have been many new adaptations to traditional cupping techniques (14):

- Moving cupping. This is a form of dry cupping that is often applied to the back (14). Practitioners use cups with a smooth edge to allow free strokes of movement. For example, bamboo cups cannot be used due to their sharp edges. Similarly, this method cannot be used on broken skin or open wounds. Although this method can be painful, it is thought to be one of the most effective forms of cupping.

- Needle cupping. This method combines both wet cupping and acupuncture. It is thought to be most beneficial for knee and elbow joints (14). After acupuncture is begun, the needles are left in position. Then a layer of oil is applied to the surrounding skin. A bamboo or glass cup is applied over the area with an acupuncture needle in place for 10 to 15 minutes.

- Herbal cupping. Bamboo cups are boiled in a large pan with a variety of herbs tailored to the patient's needs $(7,14)$. It is thought that the cups will absorb the herbal remedy, which will then be transferred to the patient. The cups are pulled out of the water one by one, heated with a flame, and then applied to the patient. The cups are left for 10 to 20 minutes. Practitioners must be very careful when using herbal cupping to avoid burns.

- Water cupping. In this type of cupping, a bamboo or glass cup is partially filled with water and the cupping procedure is carried out quickly $(7,14)$. It requires a higher level of skill to avoid spilling the water. It is thought to be beneficial in cases of asthma or coughing. It also results in minimal bruising and is often used in children.

- Pulsatile cupping. This method can be used as an adaptation to either wet or dry cupping. A pulsatile vacuum is generated through a pump mechanism $(17,19)$. It is thought to be most beneficial in treating large joints, especially for osteoarthritic pain.

\section{Understanding the mechanism}

As interest in CAM increases, many studies have attempted to explain the mechanism by which cupping may provide relief to the patient. One prospective study used dry cupping over the infraspinatus muscle, a typical location for shoulder pain, and used nearinfrared spectroscopy to assess changes in hemoglobin (20). The study showed a significant drop in hemoglobin with an increase in oxygenated hemoglobin in the cupping areas. They concluded that cupping facilitated oxygenation of muscles and thus may aid in improving muscular activity and decreasing pain.

Another study set out to understand the biological process be- hind decreased pain expression due to cupping (21). Wet cupping was used on the paralumbar region of rat models. Researchers found that heat shock protein 70 (HSP7o), a chaperone protein that prevents stress-induced denaturation, and beta-endorphin, an endogenous opioid, were elevated in the treatment group in comparison to control groups. They concluded that wet cupping might be beneficial in pain management through increased HSP70 and beta-endorphin expression.

Another study showed that wet cupping might be beneficial by removing oxidants and reducing oxidative stress (22). Venous blood and wet cupping blood samples were collected from fasting healthy volunteers. Spectrophotometry was used to analyze serum nitric oxide and malondialdehyde levels, and superoxide dismutase and myeloperoxidase activity were measured. The results showed higher levels of myeloperoxidase, malondialdehyde, and nitric oxide but lower levels of superoxide dismutase in wet cupping blood compared to venous blood. They concluded that the therapeutic effect of wet cupping may be linked to the excretion of oxidants from the body because the wet cupping sample had a higher level of oxidants compared to the venous samples (22).

\section{Cupping in dermatology}

Cupping has been used to treat various dermatological conditions (Table 1). A systematic review of eight randomized controlled trials that included 651 patients showed that wet cupping was superior to medical management in treatment of herpes zoster with regard to cure rate, improved symptoms, and reduced incidence of post-herpetic neuralgia (23). However, one report describes a secondary case of herpes simplex virus (HSV) infection secondary to acupuncture and cupping (24). The patient had been treated with both methods for myalgia of the elbow. Three days later, she developed painful, erythematous papules. The diagnosis was confirmed by skin biopsy showing the inclusion bodies and ballooning degeneration classically seen in HSV. Other known triggers to HSV reactivation include laser surgery, dermabrasion, and local tissue trauma $(25,26)$.

Table 1 | Dermatological indications for cupping.

\begin{tabular}{lc}
\hline Dermatological conditions & References \\
\hline Herpes zoster and associated post-herpetic neuralgia & $(23)$ \\
Urticaria & $(27)$ \\
Acne vulgaris & $(28,29)$ \\
Eczema & $(30,31)$ \\
Psoriasis & $(34)$ \\
Erysipelas & $(35)$ \\
\hline
\end{tabular}

$\mathrm{Li}$ and Ding reported on 40 patients treated with cupping for urticaria (27). Patients in the therapeutic arm had symptoms of urticaria lasting between 1 day and 3 years. Twenty control patients were treated with a traditional Chinese remedy and a first-generation antihistamine. The results showed significant improvement in the control group as evaluated by the Criteria for the Therapeutic Effects of Traditional Chinese Medicine on Diseases and Syndromes. In the control group, the cure rate was 55\% compared to $30 \%$ in the control group. They described the case of one patient in the treatment group, a 19-year-old Chinese female, whose urticaria was reduced to light itching after three sessions. The patient's urticaria completely disappeared after six treatments with no relapse at the 1-year follow-up.

Cupping has also been used in treating acne. In one singleblind prospective study, patients with moderate acne vulgaris received pricking bloodletting, a form of wet cupping, twice a week 
for 6 weeks (28). Acne vulgaris was evaluated using the global acne grading system (GAGS) score. The GAGS score is graded as mild, severe, and very severe. The score accounts for the following factors: area of involvement, distribution of acne, and density of pilosebaceous units on the patient's face, chest, and back. Patients showed an improved GAGS score with improvement in their acne vulgaris at the end of intervention. In another study of the treatment of acne vulgaris, wet cupping in addition to a Chinese herbal mask was compared to a control group using a Chinese herbal mask alone (29). The treatment group with wet cupping had a significant clinical improvement rate of $94.7 \%$ compared to $61.1 \%$ in the control group after a 2-week course of treatment (29). One limitation to the study was the lack of quantitative endpoints to assess the efficacy of treatment.

Yao and Li reported wet cupping as an effective treatment for acute eczema in comparison to patients treated with oral loratadine and topical ointments (30). However, Hon et al. described the case of an 11-year-old girl with high IgE levels, eczema, allergic rhinitis, and asthma (31). After many trials of diet modification, the patient's parents pursued cupping and acupuncture therapy. One day after treatment, the patient developed blistering, which progressed into two deep, exudative ulcers. The authors advocated against seeking "extreme alternative therapy" because eczema is more safely treated with topical steroids and emollients. The mainstay of treatment for atopic dermatitis is topical corticosteroids and emollients, which are often successful and safe (32). For patients with moderate to severe disease, standard treatment options currently include topical calcineurin inhibitors, phototherapy, and immunomodulators such as cyclosporine and dupilumab $(32,33)$. However, if a patient desires cupping as a supplement to the standard treatment, a trained professional should be sought.

Furthermore, cupping has been used in the treatment of psoriasis. A case report described the use of al-hijamah in treating a 30-year-old Saudi male with psoriasis (34). The patient had a Psoriasis Area Severity Index (PASI) score of 2 and had been unsuccessfully treated with topical steroid creams. After three sessions of al-hajimah, the patient had a 90\% remission of the lesions. At the 6-month follow-up, the patient had not suffered any new lesions but had minimal recurrence of previous psoriatic lesions below the elbow. The authors concluded that cupping has a potential role in treating psoriasis. However, more studies are needed, because psoriasis is a dermatological condition that can remit spontaneously.

One case series reports the successful use of combination of needling and cupping for treatment of erysipelas, an infection of the upper dermis layer. The majority of patients had erysipelas on the tibial surface (35). The authors treated 20 patients with 3 to 7 sessions of combined cupping and needling. They observed symptomatic relief in 16 patients with no clinically observed relapse. The authors advocated for the use of these techniques especially to promote better blood circulation, which may play a role in reducing inflammation. We do not advocate for the use of cupping as an alternative to antibiotic therapy; however, cupping may play a role in reduction of inflammation and pain.

Thus, it is clear that there have been attempts to apply cupping to treat a variety of dermatological conditions. However, the majority of studies and reports were carried out using a small sample size and qualitatively assessed the efficacy of treatments. More studies, and preferably randomized controlled trials, are needed to truly demonstrate the role of cupping in treating dermatological conditions.

\section{Cupping critiques and adverse events}

Although there has been a move to integrate CAM with modern medical practices, there is still high skepticism among physicians, especially in the United States (36). There are few randomized controlled trials that support the use of cupping to treat various diseases. Furthermore, many studies have been published in Chinese, making them less accessible to Western practitioners. However, physicians are also hesitant to incorporate cupping in their practice or recommend it to patients due to reports of adverse events such as bruising, skin irritation, and infection (Table 2).

Table 2 | Dermatological indications for cupping.

\begin{tabular}{lc}
\hline Adverse effects & References \\
\hline Secondary herpes simplex virus (HSV) infection & $(24-26,40)$ \\
Blistering & $(31)$ \\
Burns, bruises: possibly mistaken for child abuse & $(37,38,45)$ \\
Iron deficiency anemia & $(40-42)$ \\
Panniculitis & $(40,43,44)$ \\
Infection: possibly leading to abscess formation & $(46)$ \\
\hline
\end{tabular}

Cupping lesions have often been mistaken for child abuse (37). This is especially true in emergency rooms, where physicians are trained to be aware of signs of possible abuse in pediatric patients. Sandler and Haynes described a case of cupping mistaken for child abuse in an 11-year-old Mexican-American female (38). The patient had been seen in an emergency department for shoulder pain of 1 month's duration. The mother of the patient was not satisfied with the treatment received in the emergency room and performed dry cupping for her daughter. At follow-up 1 week later; the patient had four circular patterns consistent with firstdegree burns. The burns were likely due to the cups being placed on the skin for an extended time period. The patient's pain was still present and the family was advised against using "folk remedies." This case was not child abuse. However, it suggests that CAM practices such as cupping should be performed by trained professionals in an appropriate setting. Furthermore, it is necessary to increase cultural awareness and to take a comprehensive medical history in order to prevent false reports of child abuse (39).

One group conducted a systematic review of the literature for adverse events due to cupping (40). Anemia, panniculitis, and herpes viral infection were among the most reported adverse events due to cupping. They attributed the adverse events to unqualified personnel or patients performing the cupping and extended periods of cupping longer than 1 month. Adverse events, such as anemia, were also more likely with wet cupping.

One case report describes a 39-year-old male that presented with fatigue and dyspnea on exertion (41). The patient had undergone 6 months of wet cupping for chronic muscular pain. Labs showed iron deficiency anemia, and occult blood loss was ruled out by stool occult blood tests, esophagogastroduodenoscopy, and colonoscopy. The authors stressed the importance of considering cupping in the differential for iron deficiency anemia in patients for whom it is culturally appropriate.

Another case report describes a 66-year-old female that used wet cupping for over 10 years at home (42). She presented with fatigue, pallor, jugular vein engorgement, crackles in both lungs, and bilateral pitting edema. The patient was found to have severe iron deficiency anemia with hemoglobin of $1.5 \mathrm{~g} / \mathrm{dl}$. She was also found to have cardiomyopathy with left ventricular concentric hypertrophy. After 3 months of treatment, the patient's cardiac failure reversed with improved cardiomegaly on radiographs and 
normalized left ventricular wall thickness on echocardiogram. This case again highlights the need for cupping to be carried out by trained professionals.

There have been a few cases reported of factitial panniculitis. One case describes a 54-year-old woman that had been treated with both cupping and acupuncture for several months due to myalgia (43). The patient had several painful skin lesions on all extremities. Histopathology revealed severe fibrotic changes and hyalinization of collagen fibers with fat necrosis likely induced by trauma from cupping and acupuncture. Most such cases will resolve spontaneously; however, surgical intervention might be necessary in complicated cases (44). Another report describes factitial panniculitis in a 56-year-old woman after cupping performed by the patient herself (44). The authors stressed the importance of seeking trained professionals when using cupping therapy.

Another risk of cupping, especially when administered by untrained professionals, is burns. Iblher and Stark describe the case of a 59-year-old woman that acquired superficial to deep partial thickness burns over $15 \%$ of her body surface area (45). The patient received cupping therapy from an untrained family member. The patient underwent surgical debridement and was admitted to the inpatient unit for 10 days. Healing was further complicated by allergic reactions to various topical lotions. The authors concluded that patients should be aware of all possible complications, especially because the scientific evidence for cupping is minimal.

There have been reports of infectious adverse events after cupping procedures. One report describes a cervical epidural abscess as a complication to combined wet cupping and acupuncture administered in a Korean hospital by a trained medical professional
(46). The patient received the combined procedure for persistent headache and 1 day later was admitted due to low-grade fever, swelling and pain in the posterior neck and submandibular region. The patient responded quickly to empiric antibiotic treatment and surgical intervention was deferred. This case demonstrates the importance of sterility and the use of a hygienic environment during cupping procedures.

\section{Conclusion}

Cupping is a form of CAM that has been around for thousands of years. The practice has flourished throughout many civilizations and has recently risen to popularity. Cupping has been used to treat a variety of conditions ranging from arthritic pain to acne. Unfortunately, the majority of studies that have examined the efficacy of cupping in treating different conditions have been only small-scale. Furthermore, the majority of these studies were carried out in countries where CAM procedures are commonly integrated with standard medical treatment. Many studies have also been published in a language other than English. There is a need to conduct large-sized, randomized clinical trials to truly assess the role of cupping in dermatological conditions and possible adverse outcomes. However, the use of cupping in medicine, and specifically dermatology, is promising. Recently there has been a rise in interdisciplinary management of patient care. Many patients individually seek out CAM practices such as cupping. It is important for clinicians to be familiar with these procedures in order to properly counsel patients. Overall, cupping may play a promising role in managing patients with dermatological conditions.

\section{References}

1. Haller JS, Jr. The glass leech. Wet and dry cupping practices in the nineteenth century. N Y State J Med. 1973;4:583-92.

2. Qureshi NA, Ali GI, Abushanab TS, El-Olemy AT, Alqaed MS, El-Subai IS, et al. History of cupping (hijama): a narrative review of literature. J Integr Med. 2017; $15: 172-81$.

3. Ebeid NI. Egyptian medicine in the days of the pharaohs. Cairo: General Egyptian Book Organization; 1999.

4. Christopoulou-Aletra H, Papavramidou N. Cupping: an alternative surgical procedure used by Hippocratic physicians. J Altern Complement Med. 2008; 14:899-902.

5. Kouskoukis CE, Leider M. Cupping: the art and the value. Am J Dermatopathol 1983:5:235-40.

6. Iqbal MN, Ansari AA. Al-hijamah (cupping): the natural holistic healing art-a review. Int J Adv Ayurveda Yoga Unani Siddha Homeopathy. 2013;2:23-9.

7. Cao H, Han M, Li X, Dong S, Shang Y, Wang Q, et al. Clinical research evidence of cupping therapy in China: a systematic literature review. BMC Complement Altern Med. 2010;10:70.

8. Chirali IZ. Traditional Chinese medicine cupping therapy. London: Elsevier Churchill Livingstone; 2007.

9. AlRawi SN, Khidir A, Elnashar MS, Abdelrahim HA, Killawi AK, Hammoud MM, et al. Traditional Arabic \& Islamic medicine: validation and empirical assessment of a conceptual model in Qatar. BMC Complement Altern Med. 2017;17:157.

10. Khan MM. Sahih Al-Bukhari: Arabic-English. Gujranwala: Taleem-ul-Quran Trust; 1971.

11. Musumeci G. Could cupping therapy be used to improve sports performance? J Funct Morphol Kinesiol. 2016;1:373-7.

12. Khan HH, Khan ZH. Rio Olympics and athletes with red spots. J Pioneer Med Sci. 2016;6:127.

13. King DF, Davis MW. Cupping: an erstwhile common modality of therapy. I Am Acad Dermatol. 1983;8:563.

14. Manz A. The art of cupping. Stuttgart: Thieme; 2009.

15. Yoo SS, Tausk F. Cupping: East meets West. Int J Dermatol 2004;43:664-5.

16. Ahmadi A, Schwebel DC, Rezaei M. The efficacy of wet-cupping in the treatment of tension and migraine headache. Am J Chin Med 2008;36:37-44.

17. Mehta P, Dhapte V. Cupping therapy: a prudent remedy for a plethora of medical ailments. J Tradit Complement Med. 2015;5:127-34.
18. El Sayed S, Mahmoud H, Nabo M. Methods of wet cupping therapy (al-hijamah): in light of modern medicine and prophetic medicine. Altern Integr Med. 2013; 2:1-16.

19. Teut M, Kaiser S, Ortiz M, Roll S, Binting S, Willich SN, et al. Pulsatile dry cupping in patients with osteoarthritis of the knee: a randomized controlled exploratory trial. BMC Complement Altern Med. 2012;12:184.

20. Li T, Li Y, Lin Y, Li K. Significant and sustaining elevation of blood oxygen induced by Chinese cupping therapy as assessed by near-infrared spectroscopy. Biomed Opt Express. 2017;8:223-9.

21. Subadi I, Nugraha B, Laswati $\mathrm{H}$, Josomuljono $\mathrm{H}$. Pain relief with wet cupping therapy in rats is mediated by heat shock protein 70 and $\beta$-endorphin. Iran J Med Sci. 2017;42:384.

22. Tagi SM, Celik HT, Ciftci S, Kazanci FH, Arslan M, Erdamar N, et al. Wet-cupping removes oxidants and decreases oxidative stress. Complement Ther Med 2014; 22:1032-6.

23. Cao H, Zhu C, Liu J. Wet cupping therapy for treatment of herpes zoster: a systematic review of randomized controlled trials. Altern Ther Health Med. 2010; 16:48-54.

24. Jung Y, Kim J, Lee H, Bak H, Hong SP, Jeon SY, et al. A herpes simplex virus infection secondary to acupuncture and cupping. Ann Dermatol. 2011;23:67-9.

25. Silverman AK, Laing KF, Swanson NA, Schaberg DR. Activation of herpes simplex following dermabrasion: report of a patient successfully treated with intravenous acyclovir and brief review of the literature. J Am Acad Dermatol. 1985;13:103-8.

26. Fatahzadeh M, Schwartz RA. Human herpes simplex virus infections: epidemiology, pathogenesis, symptomatology, diagnosis, and management. J Am Acad Dermatol. 2007;57:737-63.

27. Li L, Ding J. Treatment of urticaria with cupping at back-shu points: a report of 40 cases. J Trad Chin Med. 2001;21:37-8.

28. Xu J, Lin R, Wang J, Wu Y, Wang Y, Zhang Y, et al. Effect of acupuncture anesthesia on acne vulgaris of pricking-bloodletting cupping: a single-blind randomized clinical trial. J Trad Chin Med. 2013;33:752-6.

29. Hong T, Wu L. Clinical observation on pricking bloodletting therapy at back-shu acupoints plus Chinese herbal mask in treating patients with acne. J Acupunct Tuina Sci. 2013;11:286-8. 
30. Yao J, Li NF. Clinical observation on pricking and blood-letting and cupping with a three-edge needle for treatment of acute eczema. Zhongguo Zhen Jiu. 2007; 27:424-6.

31. Hon KL, Luk DC, Leong KF, Leung AK. Cupping therapy may be harmful for eczema: a PubMed search. Case Rep Pediatr. 2013;2013:605829.

32. Eichenfield LF, Tom WL, Berger TG, Krol A, Paller AS, Schwarzenberger K, et al. Guidelines of care for the management of atopic dermatitis: section 2. Management and treatment of atopic dermatitis with topical therapies. J Am Acad Dermatol. 2014;71:116-32.

33. Beck LA, Thaçi D, Hamilton JD, Graham NM, Bieber T, Rocklin R, et al. Dupilumab treatment in adults with moderate-to-severe atopic dermatitis. N Engl J Med 2014;371:130-9.

34. Malik IA, Akhter S, Kamal MA. Treatment of psoriasis by using hijamah: a case report. Saudi J of Biol Sci. 2015;22:117-21.

35. Sang J, Wang S, Lu X. Needling and cupping used to treat 20 cases of erysipelas. J Tradit Chin Med. 2003;23:116.

36. Koo J, Arain S. Traditional Chinese medicine for the treatment of dermatologic disorders. Arch Dermatol. 1998;134:1388-93.

37. Kirschner RH, Stein RJ. The mistaken diagnosis of child abuse: a form of medical abuse? Am J Dis Child. 1985;139:873-5.
38. Sandler AP, Haynes V. Nonaccidental trauma and medical folk belief: a case of cupping. Pediatr.1978;61:921-2.

39. Patel B, Butterfield R. Common skin and bleeding disorders that can potentially masquerade as child abuse. Am J Med Genet C Semin Med. 2015;169:328-36.

40. Kim T, Kim KH, Choi J, Lee MS. Adverse events related to cupping therapy in studies conducted in Korea: a systematic review. Eur J Integr Med. 2014;6:434-40.

41. Lee HJ, Park NH, Yun HJ, Kim S, Jo DY. Cupping therapy-induced iron deficiency anemia in a healthy man. Am J Med 2008;121:e5-e6.

42. Sohn I, Jin E, Cho J, Kim C, Bae J, Moon J, et al. Bloodletting-induced cardiomyopathy: reversible cardiac hypertrophy in severe chronic anaemia from long-term bloodletting with cupping. Eur J Echocardiogr. 2008;9:585-6.

43. Lee JS, Ahn SK, Lee SH. Factitial panniculitis induced by cupping and acupuncture. Cutis. 1995;55:217-8.

44. Moon SH, Han HH, Rhie JW. Factitious panniculitis induced by cupping therapy. J Craniofac Surg. 201;22:2412-4.

45. Iblher N, Stark B. Cupping treatment and associated burn risk: a plastic surgeon's perspective. J Burn Care Res. 2007;28:355-8.

46. Lee J, Cho J, Jo D. Cervical epidural abscess after cupping and acupuncture. Complement Ther Med. 2012;20:228-31. 